\title{
Transformation of Low-Grade Mucinous Neoplasm of the Appendix With Pseudomyxoma Peritonei to High-Grade Sarcomatoid Carcinoma
}

\author{
Yu-Nung Chen ${ }^{a}$, Jie-Jen Lee ${ }^{a}$, , Shih-Ping Chenga ${ }^{a}$, Chung-Hsin Tsai ${ }^{a}$, b, c
}

\begin{abstract}
A 66-year-old man initially underwent appendectomy and cytoreductive surgery for a low-grade appendiceal mucinous neoplasm with pseudomyxoma peritonei. One and a half years later, multiple disseminated lesions developed in rectus abdominis muscle and peritoneal cavity. Biopsy showed histopathological transformation to sarcomatoid carcinoma. This case illustrates that evolution of low-grade pseudomyxoma peritonei to high-grade carcinoma truly develops in some patients. The development of this dedifferentiation appears associated with aggressive behavior and poor clinical outcome.
\end{abstract}

Keywords: Dedifferentiation; Pseudomyxoma peritonei; Recurrence

\section{Introduction}

Pseudomyxoma peritonei is a rare peritoneal disseminated disease usually arising from a primary appendiceal mucinous epithelial neoplasm [1]. It is characterized by peritoneal deposits of adenomucinous tumor cells producing a progressive amount of intraperitoneal mucinous ascites. Aggressive cytoreductive surgery with peritonectomy procedures combined with perioperative intraperitoneal chemotherapy can be curative, leading to long-term survival [2]. However, recurrent or progressive disease following treatment is not uncommon and may require repeated cytoreductive surgery that is usually less successful. Tumor biology is a major determinant of prognosis in recurrent or progressive pseudomyxoma peritonei [3].

Pseudomyxoma peritonei of appendiceal origin could be classified as low-grade and high-grade, given that high-grade

Manuscript accepted for publication April 14, 2015

${ }^{a}$ Department of Surgery, MacKay Memorial Hospital and Mackay Medical College, Taipei, Taiwan

bMackay Junior College of Medicine, Nursing, and Management, Taipei, Taiwan

${ }^{\mathrm{c} C}$ Corresponding Author: Chung-Hsin Tsai, Department of Surgery, MacKay Memorial Hospital, 92, Chung-Shan North Road, Sec 2, Taipei 10449, Taiwan. Email: surg.mmh@gmail.com

doi: http://dx.doi.org/10.14740/jocmr2178w tumor conferred a poor outcome [4]. During recurrence or progression, dedifferentiation has been reported and was shown to have a significant negative impact on survival [5]. Nonetheless, the real occurrence of dedifferentiation is controversial. It is argued that the discordant pathological findings could be a result of inadequate sampling, tumor heterogeneity, interobserver variations, and lack of uniformity in classification [6]. Here, we report an unusual case of low-grade mucinous neoplasm of the appendix associated with sarcomatoid transformation during recurrence.

\section{Case Report}

A 66-year-old man with unremarkable past medical history presented with dull abdominal pain lasting 5 days. He complained of nausea and vomiting without changes in bowel habit. Physical examination revealed diffuse tenderness and abdominal distention in the presence of shifting dullness. There was slight guarding of the abdominal wall without rebound tenderness. Laboratory data were within normal limits. Computed tomographic (CT) scan showed dilation of small bowel loops, large amount of ascites, and peritoneal thickening with coarse calcifications (Fig. 1). A jelly-like mucinous material was obtained on ultrasonography-guided abdominal paracentesis. The cytological examination was positive for malignant cells. Exploratory laparotomy revealed a ruptured mucinous lesion of the appendix with gelatinous pseudomyxoma peritonei. The patient underwent appendectomy and aggressive cytoreductive surgery with no residual macroscopic tumor (R1 cytoreduction). Pathological examination confirmed the diagnosis of low-grade appendiceal mucinous neoplasm (Fig. 2). His postoperative recovery was uneventful. The patient declined further adjuvant chemotherapy.

One and a half years later, the patient manifested a firm, non-tender abdominal wall mass. The abdominal CT scan revealed several rim-enhanced lesions with calcification over peritoneum, rectus abdominis muscle and mesentery (Fig. 3). Intraperitoneal fluid was not seen. A relaprotomy with debulking intent was performed. At exploration, carcinomatosis with a dominant tumor, measuring $8.6 \times 6.7 \times 2.5 \mathrm{~cm}$, at the left upper quadrant of the abdomen was noted. Biopsy from the peritoneal cavity, mesenteric soft tissue, and part of the tumorencased intestinal wall showed sarcomatoid carcinoma (Fig. 4). The patient died of disease 1 month after the second opera- 


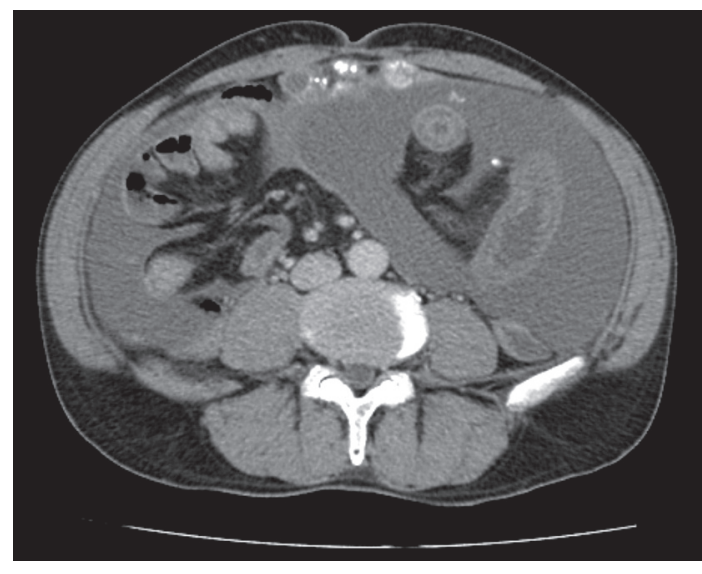

Figure 1. Abdominal computed tomographic scan before the initial operation showing massive ascites and peritoneal thickening with coarse calcifications.

tion. No autopsy was performed.

\section{Discussion}

Pseudomyxoma peritonei is a rare but intriguing disease characterized by wide spread of mucinous ascites with peritoneal and omental mucinous implants [1,7]. A mucinous epithelial neoplasm of the appendix is the most common primary tumor. As the tumor grows to occlude the lumen, mucus accumulates and the appendix eventually ruptures. The peritoneum is then seeded with mucus-producing cells, which continue to proliferate and produce mucus. The progressive accumulation of copious amounts of mucinous material gradually fills the peritoneal cavity, resulting in the characteristic "jelly belly" [8].

Histopathological classification of pseudomyxoma peritonei is complex. A generally accepted scheme proposed by Ronnett and colleagues classifies pseudomyxoma peritonei

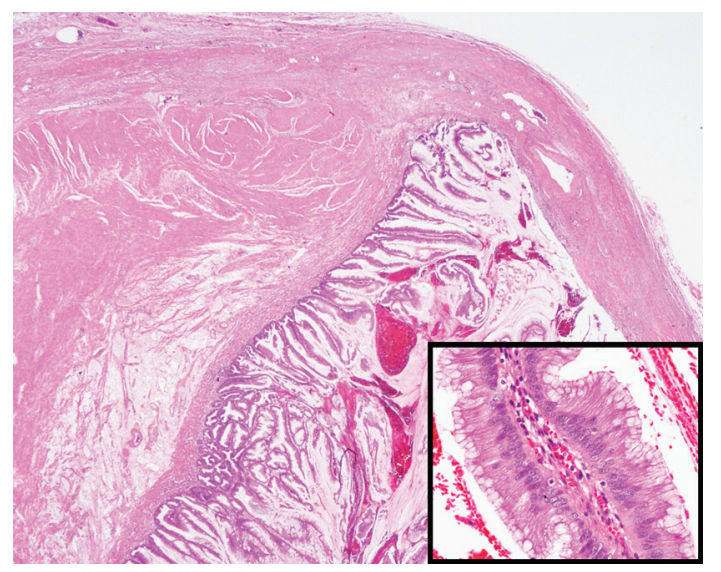

Figure 2. Microscopic appearance of the appendiceal tumor showing tall columnar epithelium with abundant extracellular mucin involving the submucosa and muscularis propria. Epithelium had no cytologic atypia or mitosis. The lumen was filled with pus and mucoid material (hematoxylin-eosin stain, original magnification, $\times 10$; inset, $\times 200$ ).

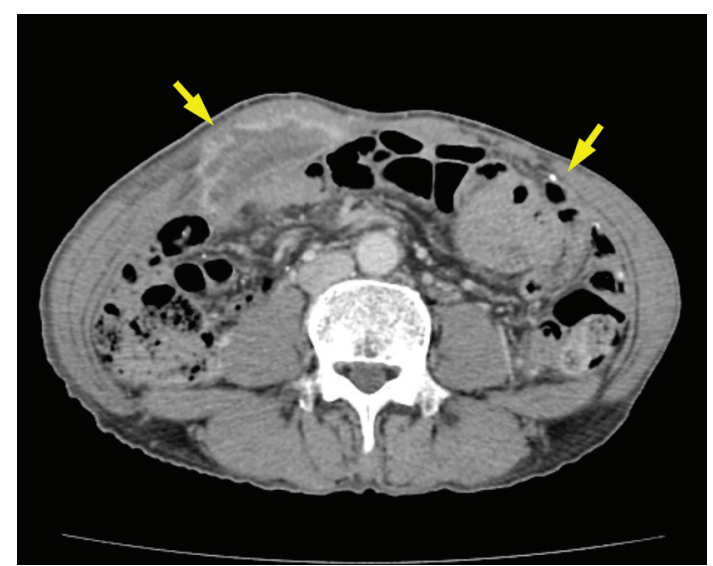

Figure 3. Abdominal computed tomographic scan after disease recurrence showing rim-enhanced lesions with calcification over rectus abdominis muscle and peritoneum (arrows).

into three subtypes with different pathological characteristics in association with different outcome: disseminated peritoneal adenomucinosis (DPAM), peritoneal mucinous carcinomatosis (PMCA), and an intermediate subtype (PMCA-I) $[9,10]$. DPAM and PMCA-I were reclassified as low-grade by Bradley and colleagues [4]. They showed that the 5-year survival for PMCA was $38 \%$, significantly worse than that for DPAM and PMCA-I (62\% and 68\%, respectively). These classifications are helpful to predict prognosis and guide therapy in patients with pseudomyxoma peritonei. Patients with low-grade disease are more likely to benefit from aggressive locoregional treatment, whereas those with high-grade disease should be treated as peritoneal carcinomatosis of colorectal origin [3, 10]. Irrespective of morphology, the presence of a high-grade component significantly worsens patient outcomes.

Combining cytoreductive surgery and perioperative intraperitoneal chemotherapy improves the survival of patients with pseudomyxoma peritonei $[2,11,12]$. Nonetheless, a high recurrence or progression rate of $40-70 \%$ has been reported [3]. Pathological subtype, the extent of peritoneal seeding and

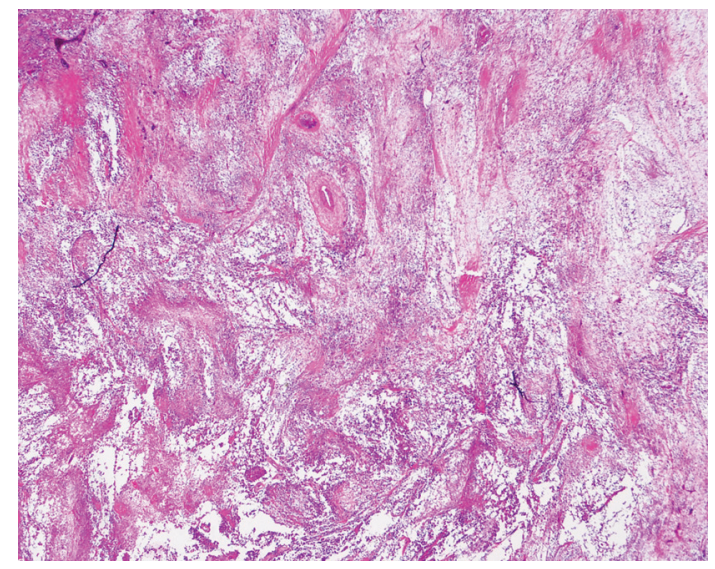

Figure 4. Biopsy of the recurrent lesions showing spindling and epithelioid pleomorphic tumor cells (hematoxylin-eosin stain, original magnification, $\times 10$ ). 
completeness of cytoreduction are the major determinants related to disease progression [3, 13]. Early recurrence following treatment failure is also indicative of a poor outcome [14]. Interestingly, male gender, as in our case, is associated with early recurrence [14].

At recurrence, low-grade disease typically retains its original low-grade histology. However, histological examination of recurrent or progressive disease may reveal a change in histopathological characteristics and biologic behavior. Pathological dedifferentiation is defined as a difference in pathology between the primary lesion and recurrent/progressive disease from DPAM towards PMCA-I or PMCA $[3,15]$. Percentages of different histology in comparison with the initial examination are described in the range of $16 \%$ to $23 \%$ of patients $[3,8$, 12]. Chua and associates reported a rate of $16 \%$ for pathological dedifferentiation in pseudomyxoma peritonei and a significant impact of this phenomenon on survival [5]. Yan and colleagues reported a series of 46 patients with pseudomyxoma peritonei who underwent cytoreductive surgery followed by a second-look operation [15]. They noted that pathological dedifferentiation occurred in $17 \%$ of patients between the first cytoreduction and the second-look operation. On the other hand, Raghav et al demonstrated that the discrepancy may result from inadequate tissue sampling [6].

Although these differences may be explained by an initial incorrect histopathological diagnosis, it is possible that evolution of low-grade neoplasm to high-grade carcinoma truly develops in some patients. The mechanism of this transformation is not certain. Recently, it has been shown that high-grade pseudomyxoma peritonei was associated with a higher frequency of p53 overexpression on immunohistochemistry [16]. Although p53 mutation is frequently observed in malignant tumors, its occurrence is particularly noteworthy among tumors showing plasticity and loss of differentiation characteristics, and p53 activation may promote differentiation in some cancers [17]. The process of pathological dedifferentiation is likely the result of the prolonged presence of disease which acquires p53 mutation at a later stage. A strategy to reduce this occurrence is to treat as early as possible and eradicate all peritoneal lesions through complete cytoreduction and sterilization of all peritoneal surface compartments with intraperitoneal chemotherapy [5].

In conclusion, transformation of low-grade appendiceal mucinous neoplasm with pseudomyxoma peritonei to highgrade sarcomatoid carcinoma is unusual, but can sometimes occur. The development of this dedifferentiation is associated with aggressive behavior and poor clinical outcome.

\section{Acknowledgement}

This work was supported by a grant from the Ministry of Science and Technology of Taiwan (MOST 103-2314-B-195-015MY3).

\section{Conflict of Interests}

The authors declare that there is no conflict of interests regard- ing the publication of this article.

\section{References}

1. Smeenk RM, Bruin SC, van Velthuysen ML, Verwaal VJ. Pseudomyxoma peritonei. Curr Probl Surg. 2008;45(8):527-575.

2. McBride $\mathrm{K}, \mathrm{McF}$ adden $\mathrm{D}$, Osler $\mathrm{T}$. Improved survival of patients with pseudomyxoma peritonei receiving intraperitoneal chemotherapy with cytoreductive surgery: a systematic review and meta-analysis. J Surg Res. 2013;183(1):246-252.

3. Smeenk RM, Verwaal VJ, Antonini N, Zoetmulder FA. Progression of pseudomyxoma peritonei after combined modality treatment: management and outcome. Ann Surg Oncol. 2007;14(2):493-499.

4. Bradley RF, Stewart JHt, Russell GB, Levine EA, Geisinger KR. Pseudomyxoma peritonei of appendiceal origin: a clinicopathologic analysis of 101 patients uniformly treated at a single institution, with literature review. Am J Surg Pathol. 2006;30(5):551-559.

5. Chua TC, Al-Zahrani A, Saxena A, Liauw W, Zhao J, Morris DL. Secondary cytoreduction and perioperative intraperitoneal chemotherapy after initial debulking of pseudomyxoma peritonei: a study of timing and the impact of malignant dedifferentiation. J Am Coll Surg. 2010;211(4):526-535.

6. Raghav KP, Taggart MW, Fournier KF, Overman MJ. Is malignant dedifferentiation for mucinous appendiceal neoplasms a valid phenomenon or merely histopathologic ambiguity? J Am Coll Surg. 2011;212(5):906; author reply 907.

7. van Ruth S, Acherman YI, van de Vijver MJ, Hart AA, Verwaal VJ, Zoetmulder FA. Pseudomyxoma peritonei: a review of 62 cases. Eur J Surg Oncol. 2003;29(8):682688.

8. Gough DB, Donohue JH, Schutt AJ, Gonchoroff N, Goellner JR, Wilson TO, Naessens JM, et al. Pseudomyxoma peritonei. Long-term patient survival with an aggressive regional approach. Ann Surg. 1994;219(2):112-119.

9. Ronnett BM, Zahn CM, Kurman RJ, Kass ME, Sugarbaker PH, Shmookler BM. Disseminated peritoneal adenomucinosis and peritoneal mucinous carcinomatosis. A clinicopathologic analysis of 109 cases with emphasis on distinguishing pathologic features, site of origin, prognosis, and relationship to "pseudomyxoma peritonei". Am J Surg Pathol. 1995;19(12):1390-1408.

10. Ronnett BM, Yan H, Kurman RJ, Shmookler BM, Wu L, Sugarbaker PH. Patients with pseudomyxoma peritonei associated with disseminated peritoneal adenomucinosis have a significantly more favorable prognosis than patients with peritoneal mucinous carcinomatosis. Cancer. 2001;92(1):85-91.

11. Sugarbaker PH. Cytoreductive surgery and peri-operative intraperitoneal chemotherapy as a curative approach to pseudomyxoma peritonei syndrome. Eur J Surg Oncol. 2001;27(3):239-243.

12. Yan TD, Black D, Savady R, Sugarbaker PH. A system- 
atic review on the efficacy of cytoreductive surgery and perioperative intraperitoneal chemotherapy for pseudomyxoma peritonei. Ann Surg Oncol. 2007;14(2):484-492.

13. Elias D, Gilly F, Quenet F, Bereder JM, Sideris L, Mansvelt B, Lorimier G, et al. Pseudomyxoma peritonei: a French multicentric study of 301 patients treated with cytoreductive surgery and intraperitoneal chemotherapy. Eur J Surg Oncol. 2010;36(5):456-462.

14. Chua TC, Liauw W, Morris DL. Early recurrence of pseudomyxoma peritonei following treatment failure of cytoreductive surgery and perioperative intraperitoneal chemotherapy is indicative of a poor survival outcome.
Int J Colorectal Dis. 2012;27(3):381-389.

15. Yan H, Pestieau SR, Shmookler BM, Sugarbaker PH. Histopathologic analysis in 46 patients with pseudomyxoma peritonei syndrome: failure versus success with a second-look operation. Mod Pathol. 2001;14(3):164-171.

16. Shetty S, Thomas P, Ramanan B, Sharma P, Govindarajan V, Loggie B. Kras mutations and p53 overexpression in pseudomyxoma peritonei: association with phenotype and prognosis. J Surg Res. 2013;180(1):97-103.

17. Spike BT, Wahl GM. p53, Stem Cells, and Reprogramming: Tumor Suppression beyond Guarding the Genome. Genes Cancer. 2011;2(4):404-419. 